\title{
Sorgfaltskriterien nicht erfüllt
}

\section{Gian Domenico Borasio}

Prof. Dr. med., Abteilung Palliativmedizin, Centre Hospitalier Universitaire Vaudois, Lausanne

Die Stellungnahme von Frau Dr. Schafroth darf man aufgrund ihrer Funktion bei Exit durchaus als «offizielle» Stellungnahme dieses Vereins betrachten. $\mathrm{Zu}$ ihrer «entschieden anderen Sicht der Dinge» möchte ich im Folgenden Stellung nehmen.

Zunächst zu der «krassen Fehlaussage», in den letzten Jahren habe eine zunehmende Anzahl von Menschen ohne körperliche Erkrankung (gemeint sind psychisch Kranke und gesunde Hochbetagte) Suizidhilfe in der Schweiz in Anspruch genommen. Für psychisch Kranke wurde mir diese Tendenz kürzlich sogar von der Geschäftsführung von Exit bestätigt, wobei richtig ist, dass die Zahlen noch sehr niedrig sind. Gesunde Hochbetagte sind bisher Einzelfälle - das dürfte sich allerdings im Zuge der von Exit angestrebten Freigabe des Alterssuizids ändern.

$\mathrm{Zu}$ den einzelnen von Frau Schafroth angesprochenen Punkten:

Ad 1.

«Sterbehilfeorganisationen [...] nehmen ihre Sorgfalts- und Fürsorgepflicht wahr, beraten umfassend und verhelfen Menschen dazu, informierte Entscheidungen zu treffen.» Nein, liebe Frau Schafroth, das tun Sterbehilfeorganisationen gerade nicht - und zwar nicht aus bösem Willen, sondern schlicht aus mangelnder Kompetenz heraus. Exits Konsiliarärzte haben in aller Regel keine psychiatrische oder palliativmedizinische Ausbildung, nehmen aber für sich in Anspruch, die Urteilsfähigkeit der Patienten fachgerecht $\mathrm{zu}$ beurteilen und ihnen eine «umfassende Beratung» anbieten zu können.

Ad 2.

Die Einschränkung der Suizidhilfe auf terminale Leidenszustände umfasst nach meinem Verständnis sehr wohl auch die schweren Fälle von Alterspolymorbidität, da diese mit einer deutlich reduzierten Lebenserwartung einhergehen. Frau Schafroth erwähnt allerdings mit keinem Wort, dass Exit auf dem Weg ist, die generelle Freigabe des Alterssuizids zu propagieren - was ganz andere, schwerwiegende gesellschaftliche Fragen aufwirft [1].

Prof. Dr. med.

Gian Domenico Borasio

Service de soins palliatifs

Centre Hospitalier

Universitaire Vaudois

Av. Pierre Decker 5

CH-1011 Lausanne

borasio[at]chuv.ch

Eine Änderung der SAMW-Leitlinien in dem von Frau Schafroth vorgeschlagenen Sinne, dass Suizidhilfe eine "freiwillige ärztliche Tätigkeit» darstellt, würde ich ebenfalls begrüssen - allerdings nur dann, wenn für deren Durchführung, wie für jede andere ärztliche Tätigkeit, klare Richtlinien und Sorgfaltskriterien festgelegt werden. Einen entsprechenden Gesetzentwurf habe ich mit drei Kollegen für Deutschland vorgeschlagen [2]. Diese Sorgfaltskriterien, das sei hier noch einmal in aller Deutlichkeit wiederholt, erfüllt die derzeitige Beratungspraxis von Exit nicht.

Ad 4.

«Menschlich-einfühlsame Begleitung» ist gut und wichtig - aber nicht ausreichend. Dass die "gut ausgebildeten Begleiter» von Sterbehilfeorganisationen (von denen die meisten nie einen Gesundheitsberuf ausgeübt haben) über die notwendigen medizinischen und therapeutischen Fachkompetenzen verfügen würden, um den Patienten entsprechend den Ursachen ihres Todeswunsches adäquate lebensorientierte Alternativen anzubieten, ist reines Wunschdenken. Dass es leider etliche Fälle gibt, in denen sich Konsiliarärzte von Schweizer Sterbehilfeorganisationen nicht einmal an die eigenen Richtlinien halten und Suizidhilfe bei Patienten durchführen, deren Urteilsfähigkeit mehr als fraglich erscheint, ist hingegen traurige Realität.

Angesichts dieser Sachlage behauptet Frau Schafroth nun, mein sehr moderater Vorschlag eines Gesetzes zur fachkundigen Beratung von Suizidwilligen würde «die persönliche Freiheit des urteilsfähigen Menschen, Art und Zeitpunkt des eigenen Todes selbst zu bestimmen, gegenüber heute gravierend einschränken». Dabei scheint sie - bei all ihrem ehrenwerten Engagement für die Selbstbestimmung - zu vergessen, dass nur wohlinformierte Entscheidungen wirklich frei und autonom erfolgen können.

Eine kurze Schlussbemerkung in eigener Sache: Ich danke Frau Kollegin Schafroth dafür, dass sie zutreffend feststellt, dass meine Position zum Thema Sterbehilfe in Deutschland als «liberal», in der Schweiz aber als eher «konservativ» betrachtet wird. Wenn man als Arzt und Wissenschaftler versucht, in einer so komplexen Debatte einen vernünftigen Mittelweg zu gehen, der sowohl die Selbstbestimmung als auch die Fürsorge berücksichtigt, ist dies kein schlechtes Zeichen.
1 Vögeli D. Alterssuizid erleichtern? Exits Spiel mit dem Feuer. NZZ 17.5.2014, online unter: www.nzz.ch/zuerich/ kommentare/1.18304153.

2 Borasio GD, Jox RJ, Taupitz J, Wiesing U. Selbstbestimmung im Sterben - Fürsorge zum Leben. Ein Gesetzesvorschlag zur Regelung des assistierten Suizids. Stuttgart: Kohlhammer-Verlag; 2014. 